\title{
USO DE NANOPARTÍCULAS DE FERRO ZERO-VALENTE NA REMEDIAÇÃO DE SOLOS CONTAMINADOS: DEGRADAÇÃO REDUTIVA DO AZUL DE METILENO
}

\author{
G. B. VIEIRA ${ }^{1}$, R. de F. P. M. MOREIRA ${ }^{2}$ e M. PETERSON ${ }^{3}$ \\ ${ }^{1}$ Universidade Federal de Santa Catarina, Programa de Pós Graduação em Engenharia Química \\ ${ }^{2}$ Universidade Federal de Santa Catarina, Departamento de Engenharia Química e Engenharia de \\ Alimentos \\ ${ }^{3}$ Universidade do Extremo Sul Catarinense, Departamento de Engenharia Química \\ E-mail para contato: gabinha24@hotmail.com
}

\begin{abstract}
RESUMO - Atualmente a nanotecnologia tem sido estudada amplamente, podendo se tornar uma das mais importantes tecnologias ambientais. Este trabalho estuda a produção de nanopartículas de ferro zero-valente (nZVI), comparando-as com nanopartículas comercializadas e sua utilização na remediação de solos contaminados com azul de metileno. As nZVI foram sintetizadas pelo método de redução do ferro férrico pelo boroidreto de sódio em condições atmosféricas, permanecendo no estado de valência zero durante dias. Para caracterizar as nanopartículas comerciais e as sintetizadas em laboratório foram realizadas análises de DRX, MET e MEV, o que resultou em partículas com diâmetro na faixa de 10-100 nm. No estudo em coluna, os resultados mostraram que as nanopartículas de ferro zero-valente influenciam no tempo de saturação da coluna pelo contaminante. O teste de adsorção em coluna teve um tempo para o início da saturação de 12 horas, os testes de degradação do corante com nZVI em coluna com 0,64\% de nZVI levou 15 horas para iniciar a saturação, a coluna com 0,96\% de nZVI levou 25 horas e para a coluna com 1,28\% de nZVI em um tempo de degradação de 45 horas ainda não havia iniciado a saturação. A degradação do corante foi confirmada pela análise de carbono orgânico total. Os resultados obtidos no estudo em coluna mostraram que o corante foi degradado pelas nanopartículas de ferro e a quantidade de nZVI influencia diretamente no processo.
\end{abstract}

\section{INTRODUÇÃO}

Com o atual crescimento populacional e o aumento constante das atividades produtivas, surgem muitas alterações ambientais em níveis extremamente preocupantes, resultando numa redução significativa da qualidade do solo, do ar e da água.

Com o objetivo de promover a correção das contaminações ambientais, muitas tecnologias são lançadas. Uma das mais importantes tecnologias ambientais disponíveis é a nanotecnologia, a qual pode ser aplicada na remediação de uma grande variedade de contaminantes, promovendo a redução 


\section{9 a 22 de outubro de 2014 \\ Florianópolis/SC}

nos custos e obtendo maiores eficiências de remediação. (KARN, KUIKEN e OTTO, 2009)

Um dos materiais nanoparticulados utilizado nesta tecnologia é o ferro zero-valente (nZVI), que tem demonstrado grandes perspectivas na degradação de compostos poluentes, principalmente os organoclorados e nitrogenados, duas classes de compostos orgânicos que, normalmente, não são eficazmente remediadas pelos processos tradicionais de tratamento de efluentes.

A degradação de compostos organoclorados através de nanopartículas de ferro zero valente representa uma tecnologia promissora para a remediação ambiental, porém as suas propriedades físicas e químicas, além da sua reatividade ainda não são inteiramente conhecidas. Surgindo a necessidade de novos estudos. (CARVALHO, 2009)

Neste contexto, este trabalho tem como proposta fazer uma abordagem dos aspectos necessários para sintetizar nanopartículas de ferro zero-valente e avaliar o processo de degradação do corante azul de metileno pelas nZVI em coluna de leito fixo.

\section{FUNDAMENTAÇÃO TEÓRICA}

Em geral, a síntese de nanopartículas pode ser feita por diversas formas, como por exemplo, pela redução da goetita ou partículas de hematita utilizando gás hidrogênio em altas temperaturas (200-600 $\left.{ }^{\circ} \mathrm{C}\right)$, pela decomposição de Ferro Pentacarbonil $\left(\mathrm{Fe}(\mathrm{CO})_{5}\right)$ em solvente orgânico ou argônio, pela eletrodeposição de sais ferrosos, entre outros (LI et al, 2006). O mecanismo mais utilizado é a redução de $\mathrm{Fe}^{+3}$ e $\mathrm{Fe}^{+2}$, como Cloreto Férrico $\left(\mathrm{FeCl}_{3}\right)$ ou Sulfato Ferroso $\left(\mathrm{FeSO}_{4}\right)$ utilizando como redutor o Boroidreto de Sódio $\left(\mathrm{NaBH}_{4}\right)$.

Avaliando os trabalhos da literatura em que os autores utilizam técnicas para sintetizar e caracterizar nanopartículas de ferro zero-valente, observa-se que a maioria deles utiliza como precursor o $\mathrm{FeCl}_{3}$ ou o $\mathrm{FeSO}_{4}$ e como agente redutor o $\mathrm{NaBH}_{4}$, obtendo partículas com diâmetro na faixa de 10-100 $\mathrm{nm}$, área superficial na faixa de $14-35 \mathrm{~m}^{2} \cdot \mathrm{g}^{-1}$ e nunca como único produto. Estes pequenos tamanhos de partículas e grandes áreas superficiais são as características mais importantes que proporcionam grande vantagem na sua utilização para remediação de águas e solos contaminados. (YUVAKKUMARA et al, 2011)

Para a produção de nanopartículas de ferro zero valente, durante a última década os custos foram reduzido drasticamente, como por exemplo, o preço caiu de US\$500/kg para US\$50-100/kg. O alto custo inicial foi devido a falta de fornecedores comerciais de nanopartículas de ferro, hoje as opções de fornecedores são múltiplas. (LI, ELLIOT e ZHANG, 2006)

As nanopartículas de ferro zero-valente são cada vez mais utilizadas na remediação de solos e aquíferos contaminados, contribuindo na recuperação do meio ambiente, porém são poucos os trabalhos encontrados sobre o estudo das nanopartículas na degradação de corantes.

Miyajima e Noubactep (2012 e 2013) publicaram dois trabalhos em que estudam o processo de degradação do corante azul de metileno pelas nZVI e pela areia. Concluíram que tanto a areia quanto 


\section{9 a 22 de outubro de 2014 \\ Florianópolis/SC}

as nZVI são eficazes na descoloração do corante, porém a presença de grandes quantidades de nanopartículas acabam prejudicando a descoloração do corante pela areia.

Btatkeu et al (2013) estudou a remoção do corante azul de metileno da areia pelas nZVI em testes de coluna de leito fixo, o qual mostrou que houve uma remoção quantitativa do corante $(>88 \%)$ e uma baixa liberação de ferro.

Os estudos demonstram claramente que a comparação dos resultados de várias fontes é difícil, mesmo quando os resultados são obtidos em condições aparentemente semelhantes. Mais pesquisas com compostos exibindo diferentes afinidades de $\mathrm{Fe}^{0} /$ areia e sobre a degradação do corante azul de metileno por nZVI em coluna contendo areia são necessárias.

\section{METODOLOGIA}

As metodologias propostas por Yuvakkumara et al (2011) e Mancilha (2006) foram utilizadas na preparação das nZVI. O método consiste em utilizar quantidades estequiométricas dos reagentes $\mathrm{FeCl}_{3}$ e $\mathrm{NaBH}_{4}$. Foi utilizada a solução de cloreto férrico $0,5 \mathrm{M}$ e de boroidreto de sódio $0,8 \mathrm{M}$ (Equação 1).

$$
4 \mathrm{Fe}^{+3}+3 \mathrm{BH}^{-4}+9 \mathrm{H}_{2} \mathrm{O} \rightarrow 4 \mathrm{Fe}^{\circ}+3 \mathrm{H}_{2} \mathrm{BO}^{-3}+12 \mathrm{H}++6 \mathrm{H}_{2}
$$

Após a preparação das soluções, titulou-se a solução de boroidreto de sódio na solução de cloreto férrico a uma vazão aproximada de 1 gota a cada 2 segundos, sob baixa agitação. Após a titulação completa, deixou-se a mistura agitando por mais 10 minutos. Em seguida filtrou-se a solução a vácuo, afim de separar o precipitado formado, o qual foi lavado com acetona para evitar a oxidação imediata do material.

As nanopartículas de ferro zero-valente foram armazenadas imersas em acetona, pois o solvente protege o material contra a oxidação, já que este pode oxidar ao primeiro contato com o ar.

Os testes em coluna de leito fixo foram realizados com objetivo de estudar o processo de adsorção do corante no solo (coluna sem nanopartículas) e a degradação do corante pelas nZVI no solo (colunas com quantidades diferentes de nanopartículas: 0,64\%, 0,96\% e 1,28\%).

Primeiramente foram preparadas as soluções do corante a uma concentração de $100 \mathrm{mg} . \mathrm{L}^{-1}$. As colunas utilizadas possuem $20 \mathrm{~cm}$ de comprimento e $3,6 \mathrm{~cm}$ de diâmetro, cada uma recheada com 280 g de areia e nZVI, dependendo do objetivo do estudo. A vazão de entrada da solução de corante foi de aproximadamente 1 gota a cada 3 segundos. Após a abertura das válvulas, as soluções de azul de metileno passam pelas colunas e amostras do efluente são coletadas nas saídas das colunas em intervalos aproximados de $20 \mathrm{~min}$. Para caracterização das amostras coletadas, foi determinada a concentração de corante (via espectrofotômetro de UV-Visível) e a concentração carbono orgânico total (via analisador de COT). 
As colunas foram contaminadas pelo corante até que a concentração de azul de metileno na saída se igualasse a concentração de entrada $\left(100 \mathrm{mg} \cdot \mathrm{L}^{-1}\right)$.

\section{RESULTADOS}

O resultado da análise de Difração de Raios-X das nZVI sintetizadas mostrou que o material obtido no processo de síntese é composto por ferro zero-valente (Figura 1). Após 20 dias, realizou-se uma nova análise de DRX com o ferro zero-valente armazenado em acetona, para verificar o seu estado de valência (Figura 2).

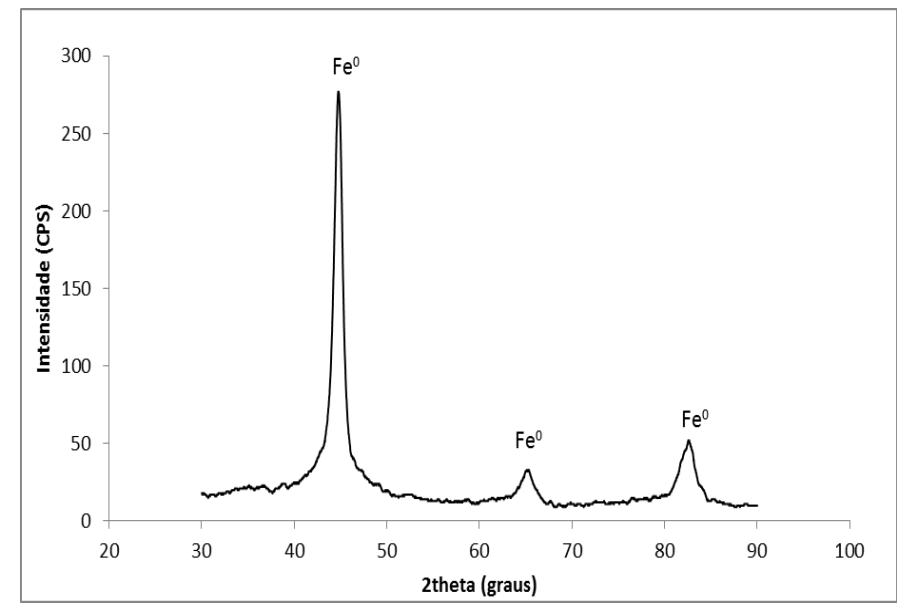

Figura 1 - Difração de Raios-X das nZVI sintetizadas.

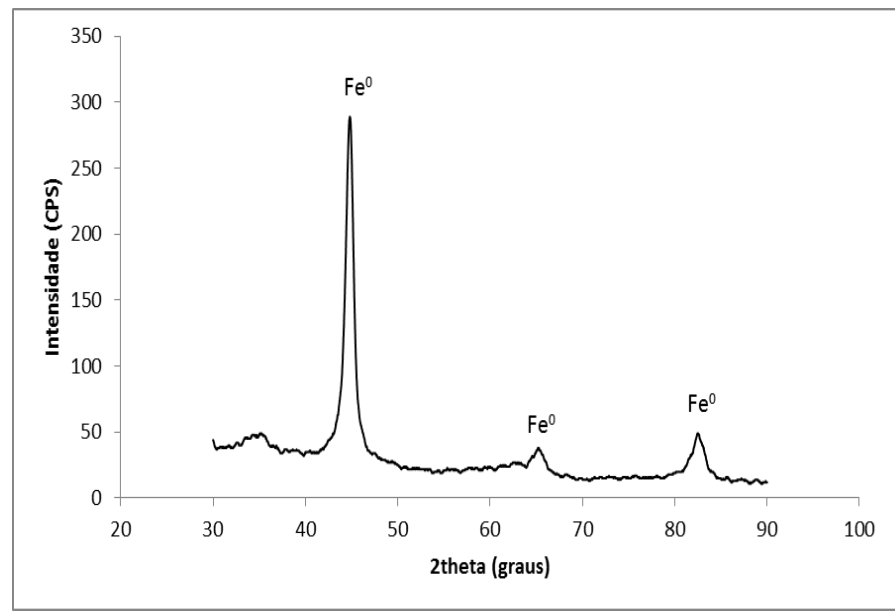

Figura 2 - Difração de Raios-X após 20 dias

A Figura 2 mostra que as nanopartículas permanecem no estado de valência zero, confirmando a eficiência da acetona na proteção do material contra a oxidação. Os picos $2 \theta=44,8^{\circ}, 65,32^{\circ} \mathrm{e}$ $82,60^{\circ}$ representam a estrutura CCC (cristalina cúbica de corpo centrado) do $\mathrm{Fe}^{0}$ (110), $\mathrm{Fe}^{0}$ (200) e $\mathrm{Fe}^{0}$ (211) respectivamente. 
Para a determinação do tamanho e avaliação da estrutura das partículas, a análise de Microscopia Eletrônica de Transmissão foi realizada (Figura 3).

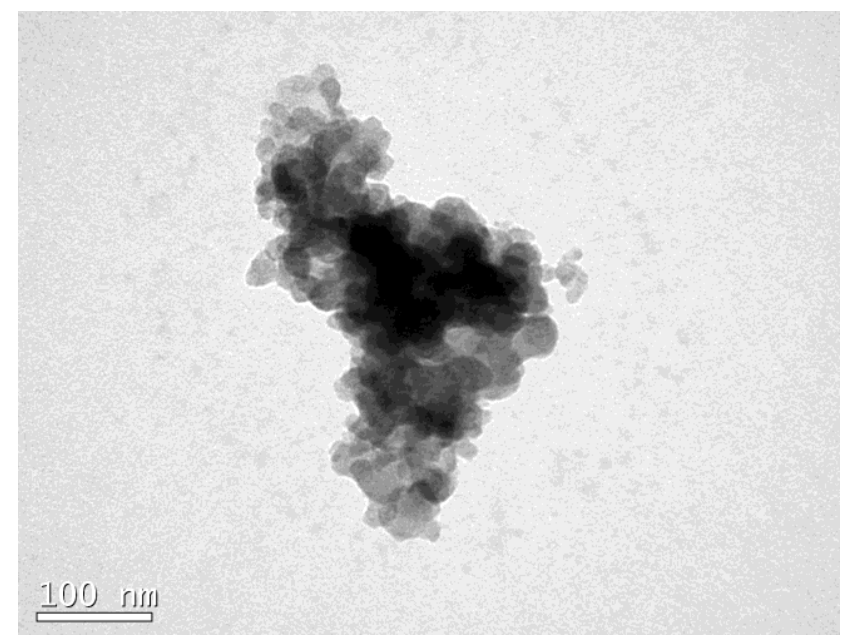

Figura 3 - Microscopia Eletrônica por Transmissão das nZVI sintetizadas.

Na Figura 3, observa-se a presença de aglomerados de partículas, esta aglomeração era esperada devido a não utilização de um estabilizante e dispersante durante a síntese. Porém, o resultado mostra que as partículas de ferro zero-valente possuem tamanho de até $100 \mathrm{~nm}$ e formato esférico.

As nanopartículas comercializadas utilizadas nos testes de degradação em coluna de leito fixo foram analisadas via Microscopia Eletrônica por Varredura (Figura 4).

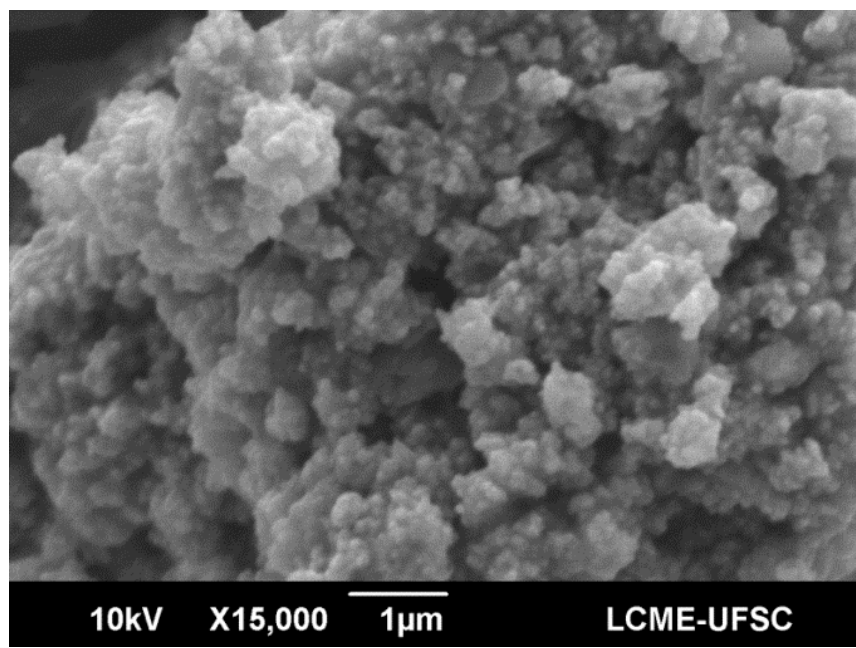

Figura 4 - Microscopia Eletrônica por Varredura das nZVI comerciais.

A Figura 4 mostra que as nZVI comerciais possuem estrutura esférica e tamanho manométrico. A aglomeração observada na imagem é devido ao processo de preparação da amostra que necessita que o material esteja seco, porém a empresa que forneceu o material utilizou um estabilizante para proteção das nanopartículas contra sedimentação e aglomeração. 
Os resultados obtidos da caracterização das nZVI comerciais podem ser comparados com os resultados obtidos da caracterização das nZVI sintetizadas em laboratório, confirmando as equivalências de tamanho e estrutura, diferenciando-as somente pela presença de aglomerados maiores nas sintetizadas em laboratório do que nas comerciais, devido a não utilização de estabilizante e dispersante.

Os testes de degradação do corante pelas nZVI em coluna de leito fixo foram realizadas em 4 colunas recheadas cada uma com $280 \mathrm{~g}$ de areia. Cada coluna recebeu uma quantidade diferente de nanopartículas. Em uma coluna foi realizado o estudo de adsorção do corante no solo, a qual não foi utilizada as nZVI. As outras três colunas receberam 0,64\% de nZVI, 0,96\% de nZVI e 1,28\% de nZVI, respectivamente, com objetivo de avaliar a influência da quantidade de nZVI na degradação do corante em coluna de leito fixo.

Durante a realização deste procedimento, coletaram-se amostras na saída de cada coluna em intervalos de tempo. Com o espectrofotômetro de UV-Visível realizou-se a leitura da absorbância de cada amostra e então foram determinadas as concentração de corante na saída da coluna de acordo com o tempo, e análise de carbono orgânico total mostrou a degradação do corante pelas nZVI. A Figura 5 mostra os resultados de concentração de corante e concentração de carbono orgânico total.

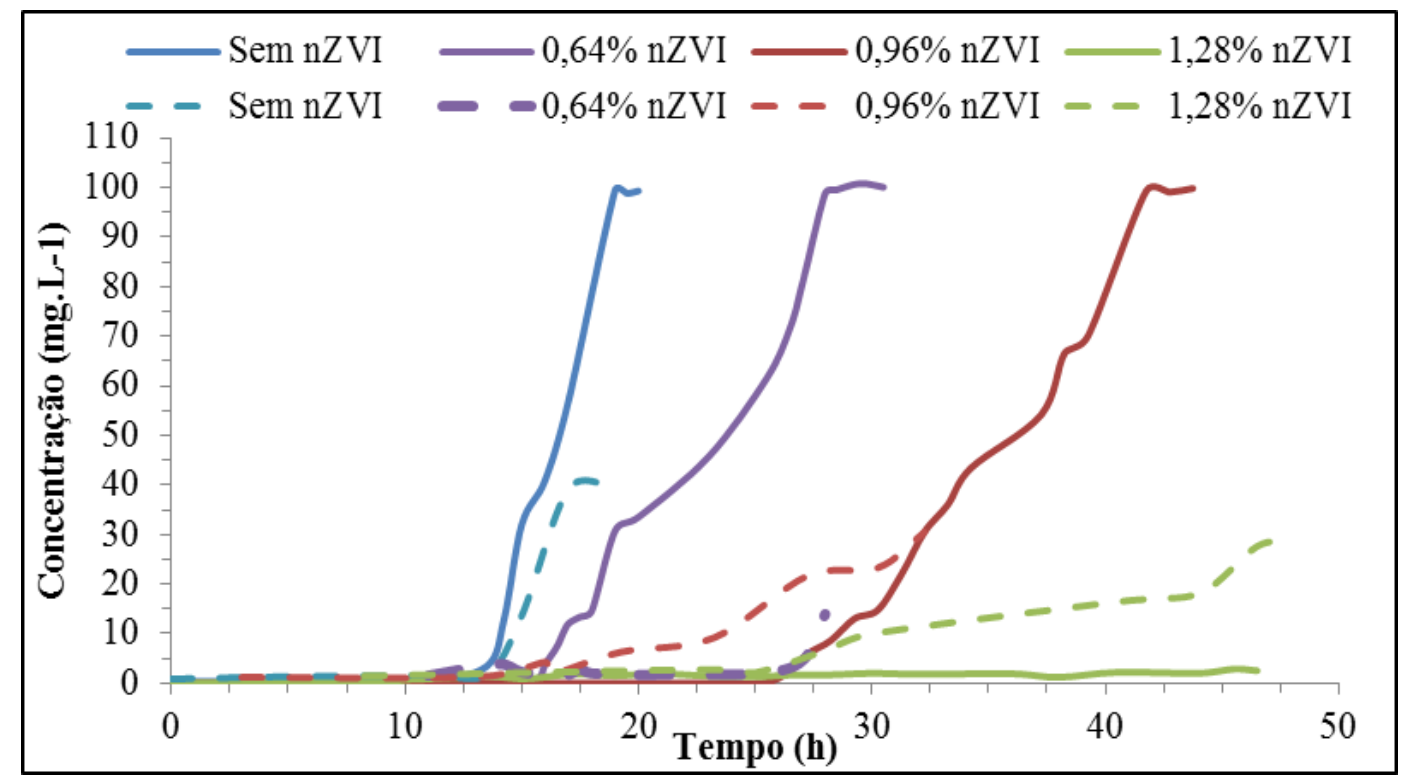

Figura 5 - Análises de COT e UV-Visível: as linhas sólidas representam as concentrações de corante e as linhas pontilhadas representam as concetrações de carbono orgânico total.

Pela Figura 5 pode-se observar que a coloração por UV-Visível não mostra se o corante está ou não sendo degradado pelas nZVI. Para a curva referente ao teste de adsorção do corante no solo em coluna (curva azul), observa-se que o aumento da concentração do corante com o tempo é equivalente ao aumento da concentração de carbono orgânico. Já para a coluna referente a degradação do corante em coluna contendo $0,64 \%$ de nZVI (curva roxa) as curvas de UV e COT se comportam de maneira diferente, ou seja, a concentração do corante na saída da coluna aumenta gradativamente conforme 
saturação da coluna, porém a concentração de carbono orgânico permanece praticamente zero até o tempo final. Isso mostra que o corante está sendo mineralizado pelas nZVI, ou seja, os carbonos orgânicos presentes foram transformados em carbonos inorgânicos. Para as colunas contendo 0,96\% e $1,28 \%$ de nZVI (curvas vermelha e verde, respectivamente), observa-se que com o aumento do tempo de degradação as concentrações de carbono orgânico total na saída das colunas aumentam mais rapidamente do que as concentrações de azul de metileno, mostrando que o corante sofreu uma modificação em sua fórmula estrutural devido a ação das nanopartículas em quebrar a dupla ligação do nitrogênio presente em sua estrutura, fazendo com que o corante passe para sua forma incolor, que é menos prejudicial ao meio ambiente.

Os resultados mostrados na Figura 5 mostra que as nanopartículas de ferro zero-valente possui uma eficiência significativa no processo de degradação do corante em coluna de leito fixo.

\section{CONCLUSÃO}

Os estudos realizados durante o processo de síntese das nanopartículas de ferro mostraram que o método empregado é de simples realização e resulta em nanopartículas com boas características, formato esférico e diâmetro na faixa 10-100 nm. Apesar da aglomeração causada pelas forças entre as partículas e a não utilização de dispersantes e estabilizante durante a síntese, os resultados estão de acordo com outros estudos presentes na literatura.

No estudo de degradação em coluna, os resultados mostraram que a presença de nanopartículas de ferro zero-valente influencia no tempo de saturação da coluna pelo contaminante, ou seja, as nZVI reagem com o corante fazendo com que o solo leve mais tempo para ficar totalmente contaminado. $\mathrm{O}$ teste de adsorção do azul de metileno no solo em coluna mostrou que o tempo para iniciar o processo de saturação da coluna foi de 12 horas. O teste de degradação do corante com nZVI em coluna de leito fixo mostrou que para a coluna contendo $0,64 \%$ de nZVI o tempo para se iniciar o processo de saturação foi de 15 horas, para a coluna com $0,96 \%$ de nZVI o tempo foi de 25 horas e para a coluna com 1,28\% de nZVI, em um tempo de degradação de 45 horas ainda não havia iniciado a saturação. Estes resultados confirmam que a quantidade de nZVI influencia diretamente sobre o tempo para contaminação do solo.

A degradação do corante pelas nZVI foi confirmada pela análise de carbono orgânico total, onde observou-se que as nanopartículas realizam dois processos de degradação: a mineralização do corante e a modificação de sua fórmula estrutural. Os dois processos resultam em produtos menos contaminantes.

O processo de degradação com a utilização de nanopartículas de ferro zero-valente mostrou-se eficiente na redução do corante azul de metileno, principalmente pelo fato de se ter utilizado quantidade pequenas de nanopartículas. 


\section{AGRADECIMENTOS}

Este trabalho é financeiramente apoiado pelo CAPES e CNPq, através do projeto. Pesquisa realizada com o apoio do LEMA e LCME-UFSC e IDT-IPARQUE-UNESC.

\section{REFERÊNCIAS}

BTATKEU, K.; MIYAJIMA, K.; NOUBACTEP, C.; CARÉ, S., 2013, "Testing the suitability of metallic iron for environmental remediation: Discoloration of methylene blue in column studies", Chemical Engineering Journal, Vol. 215-216, p. 959-968.

CARVAlHO, B. M. da S., 2009. Nanopartículas de Ferro no Tratamento de Solos Contaminados com PCBs. Dissertação de Mestrado em Engenharia do Ambiente, Universidade de Aveiro, Portugal.

KARN, B.; KUIKEN, T.; OTTO, M.; 2009, "Nanotechnology and in Situ Remediation: A Review of the Benefits and Potential Risks", Environmental Health Perspectives, Vol.117, N.12, p. 1823-1831.

LI, X.; ELLIOT, D.W.; ZHANG, W.; 2006, “Zero-Valent Iron Nanoparticles for Abatement of Environmental Pollutants: Materials and Engineering Aspects", Critical Reviews in Solid State and Materials Sciences, Vol. 31, p.111-122.

MANCILHA, J.C. Estudo do comportamento químico do ferro micro e nanoparticulado na despoluição de solos contaminados com metais pesados. São José dos Campos: INPE, 2006. $85 \mathrm{p}$.

MIYAJIMA, K.; NOUBACTEP, C. 2012. "Impact of $\mathrm{Fe}^{0}$ amendment on methylene blue discoloration by sand columns", Chemical Engineering Journal, Vol. 217, p. 310-319.

MIYAJIMA, K.; NOUBACTEP, C. 2013. "Impact of $\mathrm{Fe}^{0}$ amendment on methylene blue discoloration by sand columns", Chemical Engineering Journal, Vol. 217, p. 310-319.

YUVAKKUMARA, R.; ELANGOA, V.; RAJENDRANA, V.; KANNAN, N.; 2011, "Preparation and Characterization of zero valent iron Nanoparticles", Digest Journal of Nanomaterials and Biostructures, Vol. 6, N. 4, p. 1771-177. 